\title{
DRAGON-V: Detection and Recognition of Airplane Goals with Navigational Visualization
}

\author{
Christabel Wayllace, Sunwoo Ha, Yuchen Han, Jiaming Hu, Shayan Monadjemi, \\ William Yeoh, Alvitta Ottley \\ Washington University in St. Louis \\ St. Louis, MO 63130, USA \\ \{cwayllace, sha, hyuchen, jiaminghu, monadjemi, wyeoh, alvitta\}@wustl.edu
}

\begin{abstract}
We introduce Detection and Recognition of Airplane GOals with Navigational Visualization (DRAGON-V), a visualization system that uses probabilistic goal recognition to infer and display the most probable airport runway that a pilot is approaching. DRAGON-V is especially useful in cases of miscommunication, low visibility, or lack of airport familiarity which may result in a pilot deviating from the assigned taxiing route. The visualization system conveys relevant information, and updates according to the airplane's current geolocation. DRAGON-V aims to assist air traffic controllers in reducing incidents of runway incursions at airports.
\end{abstract}

Runway safety is of crucial importance and a constant challenge in aviation. Runway incursion refers to an incident where an unauthorized aircraft, vehicle, or person is on a runway. ${ }^{1}$ Investigations in past years found that the leading causes of runway incursion are: (1) Failure to comply with instructions provided by air traffic controllers (ATCs); (2) Lack of airport familiarity by the pilot; and (3) Non-conformance of pilots with standard operating procedures. The Federal Aviation Administration (FAA) of the United States reports that pilots caused $65 \%$ of all runway incursions. In severe cases, runway incursion can lead to tragic accidents. Therefore, aviation organizations, researchers, and inventors work on improving standards, procedures, and technology to avoid such incursions.

Researchers have proposed different types of systems to assist both pilots in the cockpit as well as ATCs. For example, Sun et al. (2019) proposed a system to predict accidents by analyzing communication errors between ATCs and pilots, and their dependency on other factors such as airport layout, time of the day, etc. Singla et al. (2019) proposed a system that alerts the aircrew and the ATC if the current state of an airplane differs from its expected state. The aircraft's current state is determined from sensors on board the airplane. (Gotteland et al. 2001) used a system based on genetic algorithms to optimize aircraft ground traffic.

Systems to avoid collisions have also been designed. Runway Awareness and Advisory Systems (RAAS) notify flight

Copyright (C) 2020, Association for the Advancement of Artificial Intelligence (www.aaai.org). All rights reserved.

${ }^{1}$ www.faa.gov/airports/runway_safety/news/runway_incursions

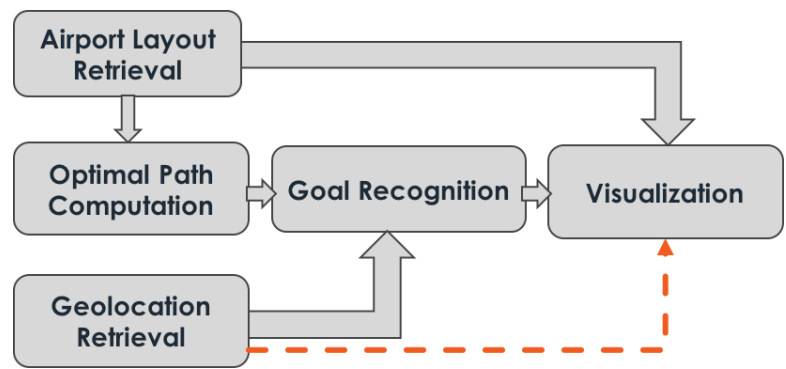

Figure 1: DRAGON-V Architecture

crews about their relative position on the ground with respect to their allocated runway (Ishihara and Johnson 2019). Other systems analyze the dynamics of the aircraft and external objects to measure collision risk (Pesik and Matty 2019) or use a radar system to detect external obstacles (Okamura et al. 2019).

In this paper, we introduce the Detection and Recognition of Airplane GOals with Navigational Visualization (DRAGON-V) system with the intention to provide a tool that could be used by the ATC to (1) Verify whether an aircraft is following its intended route; and (2) Visually determine the most probable runway(s) that the aircraft is heading towards. To the best of our knowledge, it is the first system that provides such a visual aid to the ATC. Further, DRAGON-V requires only a fairly limited set of inputs - the airport layout and current location of the airplane - which are readily available to ATCs, thereby simplifying the use and adoption of such a system in practice.

\section{DRAGON-V Architecture}

Figure 1 shows the system architecture of DRAGON-V. We describe each module below.

Airport Layout Retrieval: We retrieved the airport layout from the flight simulator X-Plane, ${ }^{2}$ where taxi and truck route networks, as well as runways, are represented as a directed graph. Nodes are described as 〈latitude, longitude〉

\footnotetext{
${ }^{2}$ https://developer.x-plane.com/article/ airport-data-apt-dat-file-format-specification/
} 


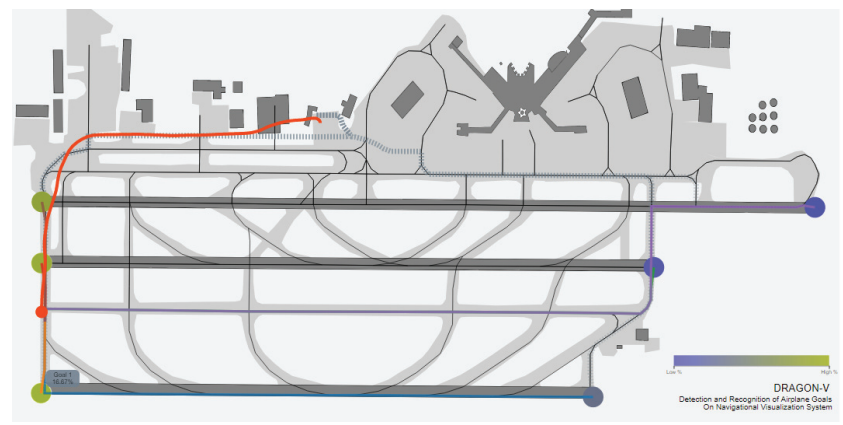

Figure 2: User Interface of DRAGON-V

pairs. We assigned costs proportional to the distance between nodes to all edges.

Optimal Path Computation: We identify nodes that correspond to runways as possible goals of pilots, and then compute the optimal paths from all nodes to all goals.

Geolocation Retrieval: The airplane's current position is obtained from X-Plane using the X-Plane Connect toolbox, ${ }^{3}$ which sends the information through UDP messages.

Goal Recognition: We use a single-observation costbased goal recognition (Masters and Sardina 2018) to rank goals according to their probability of being the goal that the pilot is trying to reach. Using their single-observation formula, we find a cost difference that assumes that the pilot is rational. However, this rationality is bounded by constraints that may affect a pilot's performance, such as limited knowledge of their environment, partial observability, etc. Similar to Ramírez and Geffner (2010), posterior probabilities are computed using Bayes' rule where the likelihood is approximated as a sigmoid function of the cost difference.

Visualization Interface: Figure 2 shows the visualization interface, where the trace of a airplane's trajectory is shown in orange. Goals are identified by circles at the extreme end of each runway and are colored according to their probability of being the true goal - high-probability maps to green and low-probability maps to purple. Optimal paths from the starting point to each possible goal are shown in dashed gray lines. The layout of the airport (Seattle-Tacoma) is shown together with the edges of the graph. A subset of those edges represent the predicted optimal paths, and are highlighted while the aircraft is moving. The current implementation stores the traversed trajectory to visualize it, however, we plan to visualize the information in an online fashion, that is, to update the visualization as new data from the airplane is received (represented in Figure 1 with the dashed orange arrow). A user can also interact with the system by hovering over a possible goal (which then displays the computed probability of the goal) or over the original optimal paths (which then shows the goal for which that path is optimal).

\footnotetext{
${ }^{3}$ https://github.com/nasa/XPlaneConnect
}

We tested DRAGON-V with different paths of increasing degree of suboptimality. Unlike other types of goal recognizers, where all observations are taken into account to determine the most probable goal (causing them to get lost with loopy trajectories), DRAGON-V will always provide ranked goals because only the current observation and the starting position are taken into account. However, this same characteristic causes the system to never be certain about one goal, even when the pilot arrives at it. The system assumes that it is possible that one goal is on the route to another.

\section{Conclusions and Future Work}

We presented DRAGON-V, a simple yet useful system designed to help ATCs detect pilot deviations and provide a ranking of possible goals of pilots. Additionally, this system could also be useful in pilot training programs to study how well they follow instructions. In future work, we plan to implement online visualizations, extend DRAGON-V to recognize the goals of multiple aircraft, and consider more complex cost functions that account for factors such as weather, congestion, risk, and condition of the route.

\section{Acknowledgments}

We would like to thank the reviewers for their constructive comments and suggestions. This research is partially supported by Boeing under award 2018-BRT-PA-332 and by NSF under awards 1812619 and 1838364.

\section{References}

Gotteland, J.-B.; Durand, N.; Alliot, J.-M.; and Page, E. 2001. Aircraft ground traffic optimization. In Proceedings of USA/Europe ATM R\&D Seminar.

Ishihara, Y., and Johnson, S. 2019. Aircraft systems and methods for managing runway awareness and advisory system (raas) callouts. US Patent App. 10/204,523.

Masters, P., and Sardina, S. 2018. Cost-based goal recognition for the path-planning domain. In Proceedings of IJCAI, 5329-5333.

Okamura, S.; Hatakeyama, T.; Yamaguchi, T.; and Uenoyama, T. 2019. Radar detection system and radar detection method. US Patent App. 15/901,102.

Pesik, J. T., and Matty, D. 2019. Determination of collision risks between a taxiing aircraft and objects external to the taxiing aircraft. US Patent App. 15/815,654.

Ramírez, M., and Geffner, H. 2010. Probabilistic plan recognition using off-the-shelf classical planners. In Proceedings of AAAI, 1121-1126.

Singla, A.; Gonabal, S. D.; Huncha, P.; Rallabandi, V.; Singh, J.; KS, S. K.; et al. 2019. System and method for monitoring compliance with air traffic control instructions. US Patent App. 15/790,220.

Sun, Z.; Zhang, C.; Tang, P.; Wang, Y.; and Liu, Y. 2019. Bayesian network modeling of airport runway incursion occurring processes for predictive accident control. In $\mathrm{Ad}$ vances in Informatics and Computing in Civil and Construction Engineering. Springer. 669-676. 